\title{
La selección de personal como contribuyente causal del desempeño laboral, un estudio explicativo realizado a docentes universitarios de la república del Ecuador.
}

The selection of staff as a taxpayer's causal work performance, an explanatory study carried out at the university teachers of the Republic of Ecuador.

Juan Gabriel Saltos Cruz. ${ }^{1}$, Lucila Alejandra Reyes Terán. ${ }^{2} \&$ Juan Carlos Reyes Terán ${ }^{3}$

Recibido: 09-12-2017 / Revisado:177-02-2018 Aceptado: 14-03-2018/ Publicado: 01-04-2018

\begin{abstract}
.
DOI: https://doi.org/10.33262/cienciadigital.v2i2.129

In a globalized world and new trends, the management of companies or organizations must be highly competitive, therefore, human capital is an important factor within the company because it generates efficiency and quality. That is to say, a human resource planning and a personnel recruitment process should be considered that promote the entry of highly qualified personnel and that efficiently develop their skills and attitudes in the position. The purpose of this paper is to validate the recruitment and selection mechanisms used in higher education for the hiring of academic staff, in order to estimate if there is causality between the categories of personnel selection and job performance.
\end{abstract}

Keywords: Organizational Commitment, Productivity, Performance, Work Environment.

\section{Resumen.}

En un mundo globalizado y las nuevas tendencias, hace que el manejo de las empresas $\mathrm{u}$ organizaciones deban ser altamente competitivas, por lo tanto, el capital humano es un factor importante dentro de la empresa ya que genera eficiencia y calidad. Es decir, se debe considerar una planeación de recursos humanos y un proceso de reclutamiento

\footnotetext{
${ }^{1}$ Universidad Técnica de Ambato, Ambato, Ecuador, jg.saltos@uta.edu.ec

${ }^{2}$ Universidad Técnica de Ambato, Ambato, Ecuador, lucecitashine@gmail.com

${ }^{3}$ Escuela Técnica de la Fuerza Aérea, Cotopaxi Ecuador, etfa33146@hotmail.com
} 
de personal que promuevan el ingreso de personal altamente calificado y que desarrollen de forma eficiente sus habilidades y actitudes en el cargo. El presente trabajo tiene como propósito validar los mecanismos de reclutamiento y selección usados en educación superior para la contratación del personal académico, a fin de estimar si existe causalidad entre las categorías de la selección del personal y el desempeño laboral.

Palabras Claves: Selección por Méritos, Selección por Oposición, Competencia.

\section{Introducción.}

La capacidad de atraer y retener a los mejores talentos se está convirtiendo rápidamente en un tema clave para la gestión de los recursos humanos en las universidades (Huisman, Weert, y Bartelse, 2002; Metcalf, Rolfe, Stevens, y Weale, 2005). Las universidades ciertamente no son los únicos empleadores que compiten por empleados altamente calificados, y un grupo de encuestas recientes sugieren que las disciplinas académicas ya están sufriendo de una escasez crónica de personas talentosas en algunos países (Gilliot, Overlaet, y Verdin, 2002; Van Balen y Van den Besselaar, 2007; Lubbe y Larsen, 2008; Edwards y Smith, 2010).

La composición y la calidad del personal académico son de vital importancia para la calidad de los programas de educación y la investigación universitaria, así como la reputación y la posición competitiva de las universidades e instituciones en la comunidad académica (Lorange, 2006). La gestión del talento y el rendimiento, por lo tanto, ha entrado en la agenda estratégica de gestión de recursos humanos de muchas universidades y se ve reforzada por la tendencia de las universidades a pasar de un modelo colegiado a uno de gestión (Deem, 2001)

La literatura actual sobre la gestión del talento y los indicadores de desempeño rara vez presta atención al proceso de implementación, los procesos de poder y el contexto (Watson, 2004; McKenna, Richardson, y Manroop, 2010). Este artículo llena este vacío estudiando las prácticas de reclutamiento y selección de académicos júnior y sénior en Holanda en la transición actual de talento académico y gestión del desempeño y, como se dijo anteriormente, reflexionando sobre tres desafíos que los gerentes de talento humano enfrentan implantan sistemas de desempeño en el reclutamiento académico y selección.

El reclutamiento y selección constituye una de las áreas más antiguas en el campo de la psicología aplicada. Además, es uno de los dominios más importantes de la gestión del talento y los recursos humanos, atractivo para un gran número de investigadores y profesionales de recursos humanos (Ryan y Ployhart, 2014). Los tiempos están cambiando y el área de reclutamiento y selección también está evolucionando rápidamente, impulsada por cuestiones estratégicas, presiones sociales y desarrollos tecnológicos. 
Dada la llamada 'guerra' por el talento en las organizaciones.

Durante las últimas décadas en Europa y más allá, el reclutamiento y selección incluso ha tomado un lugar más central en la estrategia de la organización y la gestión del talento en particular. El papel estratégico que el reclutamiento y la selección desempeñan en las organizaciones ha desembocado una serie de nuevos desarrollos y ha ampliado las conceptualizaciones tradicionales de las prácticas de investigación y desarrollo con conocimientos de otras disciplinas, como economía y mercadotecnia (Yu y Cable, 2012), información, comunicación e informática e incluso biología. Considere, por ejemplo, "juegos de reclutamiento" (Marsh, 2011) o juegos serios que se presentan como un tipo híbrido de herramientas de reclutamiento y prueba.

Estas nuevas herramientas aumentan la eficiencia atrayendo y seleccionando simultáneamente a los solicitantes al principio del ciclo de contratación, creando así ciclos de contratación y socialización más comprimidos para las personas y las organizaciones. Al mismo tiempo, los juegos de reclutamiento de alta tecnología tienen una intención de mercadotecnia ya que pueden marcar la imagen de la organización, ayudar con las relaciones públicas y posicionar estratégicamente a la organización en el mercado de trabajo local (Huisman, Weert, y Bartelse, 2002).

Aparte de cualquier impulso estratégico, la diversidad social los desafíos en Europa también impulsan las prácticas de investigación y desarrollo. Uno de los impulsores más desafiantes y apremiantes son los cambios demográficos actuales, debido a las olas migratorias que actualmente no se ven en Europa, que a su debido tiempo pueden crear una sociedad aún más compleja y un mercado laboral diverso (Huisman, Weert, y Bartelse, 2002).

En efecto, dadas estas dinámicas, surge la duda sobre la evaluación de los grupos minoritarios (con respecto a la edad, el género, etnicidad, discapacidades). Se augura que será aún más importante en los próximos años y continuará desafiando modelos o herramientas de personal bien establecidos. Además, se ajustaran a las políticas de recursos humanos centradas en occidente y que piensen en los trabajadores talentosos. Finalmente, uno de los mayores cambios en las prácticas de investigación y desarrollo es el vinculado a la tecnología (Watson, 2004).

De hecho, los avances tecnológicos redefinen las prácticas de investigación y desarrollo mediante herramientas multimedia (como pruebas móviles, servicios de video, uso de plataformas de reclutamiento, etc.). El uso de sistemas de seguimiento de candidatos en línea (como parte de los sistemas de información de recursos humanos) podría aumentar la eficiencia del proceso de reclutamiento y evaluación tanto para los reclutadores como para los solicitantes (Lorange, 2006).

Aunque algunas aplicaciones multimedia, como los sitios web de redes sociales como (Kluemper, Davison, Cao, y Wu, Roth, Bobko, Van Iddekinge y Thatcher). Además de las 
pruebas sin supervisión / móviles (por ejemplo, Burke, Mahoney-Philips, Bowler, Y Downey). Son cada vez más utilizados por solicitantes y practicantes. La investigación está un poco rezagada ya que todavía no se sabe mucho sobre las propiedades (como la seguridad de los materiales de prueba) y la eficacia (como la validez de la prueba) de varias evaluaciones mejoradas, herramientas de reclutamiento y evaluaciones más tradicionales (Gilliot, Overlaet, y Verdin, 2002).

Sin embargo, a pesar de las llamadas perdurables de prueba, existe el riesgo de que los investigadores y los profesionales se distancien en lugar de reunirse para abordar nuevos desafíos. Gran parte del debate sobre la división entre investigación y práctica nos recuerda la parábola india de los ciegos y el elefante: al examinar diferentes partes del elefante, y en virtud de la discapacidad física de uno, uno no podría ver el todo de sus partes (Gilliot, Overlaet, y Verdin, 2002).

La implementación real de los procesos de gestión del talento y el rendimiento apenas se ha estudiado. Las pocas excepciones (Hiltrop, 1999; Holland, Sheehan, y De Cieri, 2007) utilizan un enfoque más bien funcionalista y prescriptivo, que se centra en las relaciones causa-efecto, las pruebas estadísticas y el pensamiento lineal (Latham, Almost, y Moore, 2005). Sin buscar devaluar el conocimiento adquirido en estos estudios, esta investigación se adhiere a (McKenna, Richardson, y Manroop, 2010) que afirman que un aumento en la diversidad paradigmática podría contribuir a la comprensión de cómo se implantan el rendimiento académico y la evaluación. Esto implicaría pasar de un enfoque en

"Descubriendo las mejores prácticas" hacia un enfoque de investigación más crítico y reflexivo, que tenga en cuenta situaciones de poder y control y el contexto en el que se lleva a cabo la evaluación (Watson T., 2010)

Además, los modelos tradicionales de sistemas de rendimiento no han prestado suficiente atención al contexto organizacional en el que ocurren las evaluaciones. (Janssens y Steyaert, 2009) Sugieren un enfoque reflexivo basado en la práctica para la gestión de recursos humanos que tiene en cuenta múltiples partes interesadas, y un contexto moral, social y político más amplio. Hacia un enfoque de práctica contextual, este enfoque considera lo que recientemente se ha denominado la reactivación del "giro de la práctica" en los estudios organizacionales (Schatzki, Knorr Cetina, y Von Savigny, 2001). Dentro de este contexto, el enfoque analítico se centra en la organización o prácticas de trabajo. Dicho de otro modo, lo que dice la gente y lo hacen en su interacción social dentro de las organizaciones (Yanow, 2006). Tal enfoque significa que los conceptos pueden evolucionar a partir de experiencias vividas de académicos en lugar de estudios objetivistas que a menudo están separados de las experiencias y contextos tanto del trabajador como del investigador.

Se utiliza una amplia conceptualización de las prácticas de gestión de recursos humanos que incluye prácticas discursivas articuladas en documentos de política o protocolos de 
reclutamiento, así como reflexiones personales sobre cómo los académicos reclutan y seleccionan candidatos. La perspectiva de la práctica es, por lo tanto, útil para describir la interacción compleja y dinámica entre las políticas formales de las organizaciones y la práctica diaria real en el reclutamiento y la selección. Esto puede ayudarnos a entender cómo los miembros de la organización usan activamente, se resisten o alteran las normas y las políticas formales disponibles.

Como sostenemos que el reclutamiento y la selección académica no ocurren en el vacío, enfatizamos la necesidad de un enfoque que considere el contexto pertinente (ínter) organizacional. Se esperan algunas diferencias entre las prácticas de contratación y selección en los subcampos académicos, ya que cada subcampo exhibe características homólogas a una estructura social más amplia, así como a su propia estructura y lógica específicas (Deem, 2001).

Las investigaciones también han demostrado que los contextos académicos dan forma a las prácticas y procesos organizacionales, como el reclutamiento y la selección. Esta investigación se basa en la operalización del contexto académico de (Edwards y Smith, 2010) para analizar las diferencias observadas y seleccionar los aspectos que pueden relacionarse con el reclutamiento y la selección.

Hiltrop (1999) Alientan a los académicos de Gestión de Recursos Humanos a adoptar una visión más reflexiva y orientada a la práctica de Gestión de Recursos Humanos, para examinarla como un conjunto de prácticas, integradas en un contexto global, económico, político y sociocultural. Por lo tanto, consideraremos el reclutamiento y la selección como un conjunto de prácticas sociales y culturales que tienen una naturaleza claramente práctica pero que también son un campo en el que se aplican valores y se establecen conflictos entre valores e identidades grupales (Gilliot, Overlaet, y Verdin, 2002; Hiltrop, 1999). Incluso en la universidad "gerencialista" donde la bibliometría se usa para evaluar a los académicos, el reclutamiento y la selección de talentos académicos no es simplemente una tarea técnica que implica juzgar qué académicos son los mejores. También es un esfuerzo político que involucra negociaciones entre múltiples actores.

\section{Materiales y métodos}

Para el presente proyecto se usó el método empírico de recolección de información. En tal virtud la unidad de análisis de interés del investigador es: la existencia de relación o asociación entre la variable selección y rendimiento laboral. Para esto, se diseñó o codificó un sistema categorial a partir de un común denominador hallado en los presupuestos teóricos. El resultado del método hipotético deductivo da en sí una hipótesis de trabajo expresada como: la existencia de relación/asociación entre la variable selección y el rendimiento laboral. La variable exógena selección de personal será medida a partir de un instrumento estructurado (cuestionario), producto del deductivo del sistema categorial. La variable 
dependiente endógena será medida dentro del mismo instrumento y validada gracias a los departamentos de talento humano de las instituciones de educación de superior, colaboradores para este estudio.

Población, es el conjunto de datos de los cuales se ocupa un determinado estudio estadístico. La población del presente trabajo de investigación está conformada por 2703 docentes que laboran en las universidades de la provincia de Tungurahua, 1246 docentes son hombres que representa el $35 \%$ y 1457 son mujeres lo que corresponde al 65\% de la población, en esta totalidad están incluidas las personas de planta y a contratos. Información proporcionada por los departamentos de Talento Humano de las universidades participantes.

La Muestra, es un subconjunto de la población, preferiblemente representativo de la misma. Para extraer el tamaño de la muestra se tomó la población de colaboradores y para calcular el tamaño de la muestra se debe aplicar la fórmula para muestras cualitativas finitas. Además, se trabajó con 352 como muestra base, con un 28,98\% de tasa de respuesta. En cuanto al método muestral, se escogió el muestreo aleatorio simple que consiste en seleccionar una muestra de la población a partir de mecanismos de selección con criterios de aleatoriedad. Es decir, los individuos empleados en la investigación se seleccionan de un marco muestral definido, que obedece a un criterio estadístico. Esta conveniencia, que se suele traducir en una gran facilidad operativa y en bajos costes de muestreo, tiene como consecuencia la imposibilidad de hacer afirmaciones generales con rigor estadístico sobre la población.

La métrica usada fue la de intervalo que fue sujeta a una escala de Likert para la aplicación estadística. Las técnicas e instrumentos de investigación que se utilizaron: encuestas y cuestionarios. Por medio de la investigación de campo se recogió varios criterios sobre sus propias calificaciones de las categorías de merecimientos y oposición, relacionado al estado actual. Para desarrollar, sustentar y profundizar el estudio se usó el análisis de factores a través del método de máxima verosimilitud tomando en cuenta el número de factores que explica la varianza acumulada. Finalmente, se usó el método de rotación a través de Varimax para identificar la clasificación de los ítems en las categorías estudiadas.

Investigación explicativa: trata de descubrir, establecer y explicar las relaciones causales funcionales que existen entre las variables estudiadas, y sirve para explicar cómo, cuándo, dónde y por qué ocurre un fenómeno social. Los elementos de la unidad de observación fueron docentes de instituciones de educación superior de la provincia de Tungurahua, se consideró una muestra inicial calculada de 352 casos con una muestra final de 102, como características a considerar los docentes, son con nombramiento y contrato pertenecientes a instituciones privadas y públicas.

Una vez depurada la data se sometió a un análisis factorial exploratorio con criterio de máxima verosimilitud. El procedimiento fue escogido sobre la base de la necesidad planteada de un procedimiento de alto rigor. El proceso de componentes principales fue descartado por 
su grado bajo de discriminación al momento de relacionar las categorías del constructo (variables observables). Para extraer las cargas factoriales de la relación del modelo o constructo se aplicó el procedimiento Varimax que aumenta la varianza a su más alta expresión. Mediante la rotación del modelo se muestran las cargas eficientes de la relaciónasociación de las variables latentes de tipo endógena y exógena.

Para visualizar la influencia de las dos variables exógenas (Selección por Méritos y Selección por oposición) sobre la variable endógena (Rendimiento laboral) se usó una aplicación informática para el manejo de ecuaciones estructurales. Lisrel es un paquete estadístico que posibilita el cálculo de relación endógena exógena de variables sobre un modelo. Las bondades de este programa son la versatilidad para seleccionar las variables latentes, observables, endógenas y exógenas.

\section{Resultados.}

Análisis de fiabilidad.

El estudio fue realizado con 102 docentes de planta que participaron en los últimos 2 años en concursos para ingresar a instituciones de educación superior. Al momento de la depuración de la base de datos se evidencia que no existe perdida de información en ninguna de las mediciones tomadas bajo el instrumento.

Tabla No1. Resumen de procesamiento de casos

\begin{tabular}{rlrr}
\hline & & $\mathrm{N}$ & $\%$ \\
\hline \multirow{3}{*}{ Casos } & Válido $^{*}$ & 102 & 100 \\
& Excluido $^{\mathrm{a}}$ & 0 & 0 \\
& Total & 102 & 100 \\
\hline
\end{tabular}

La eliminación por lista se basa en todas las variables del procedimiento.

Para el presente caso se estudió la fiabilidad en un solo cálculo, ya que las tres variables latentes se midieron en el mismo Test. Los resultados presentan un grado de fiabilidad de medida del 88,2\% que está por sobre el estándar aceptable para este tipo de estudios, se presentan a continuación el extracto del cálculo:

Tabla No2. Estadísticas de fiabilidad

Alfa de Cronbach $\mathrm{N}$ de elementos

0,882

Análisis factorial exploratorio. 
$\mathrm{El}$ análisis de factores desde la perspectiva exploratoria empieza por estudiar los indicadores claves. Como primer estadio, la prueba Kaiser-Meyer-Olkin de adecuación de muestreo da un número muy favorable de 0,828. En cuanto a la prueba de esfericidad de Bartlett, evalúa la pertinencia de la aplicación en las variables estudiadas, que para el caso muestra un Chicuadrado mayor que 1 , además tiene una medida significativa menor de 0,05.

Tabla No 3 Prueba de KMO y Bartlett

\begin{tabular}{llr} 
Medida Kaiser-Meyer-Olkin de adecuación de muestreo & 0,828 \\
\hline & Aprox. Chi-cuadrado & 2386,93 \\
Prueba de esfericidad de Bartlett & gl & 105 \\
& Sig. & 0 \\
\hline
\end{tabular}

Como segundo estadio, el análisis de la varianza explicada da indicios de la existencia de 3 grupos de variables observadas o 3 variables latentes compuestas por los ítems estudiados, las medidas de la varianza acumulada devuelven un valor de 88,38\% de descripción de comportamiento del modelo a partir de la medida de la varianza como se muestra a continuación.

Tabla No. 4 Varianza total explicada

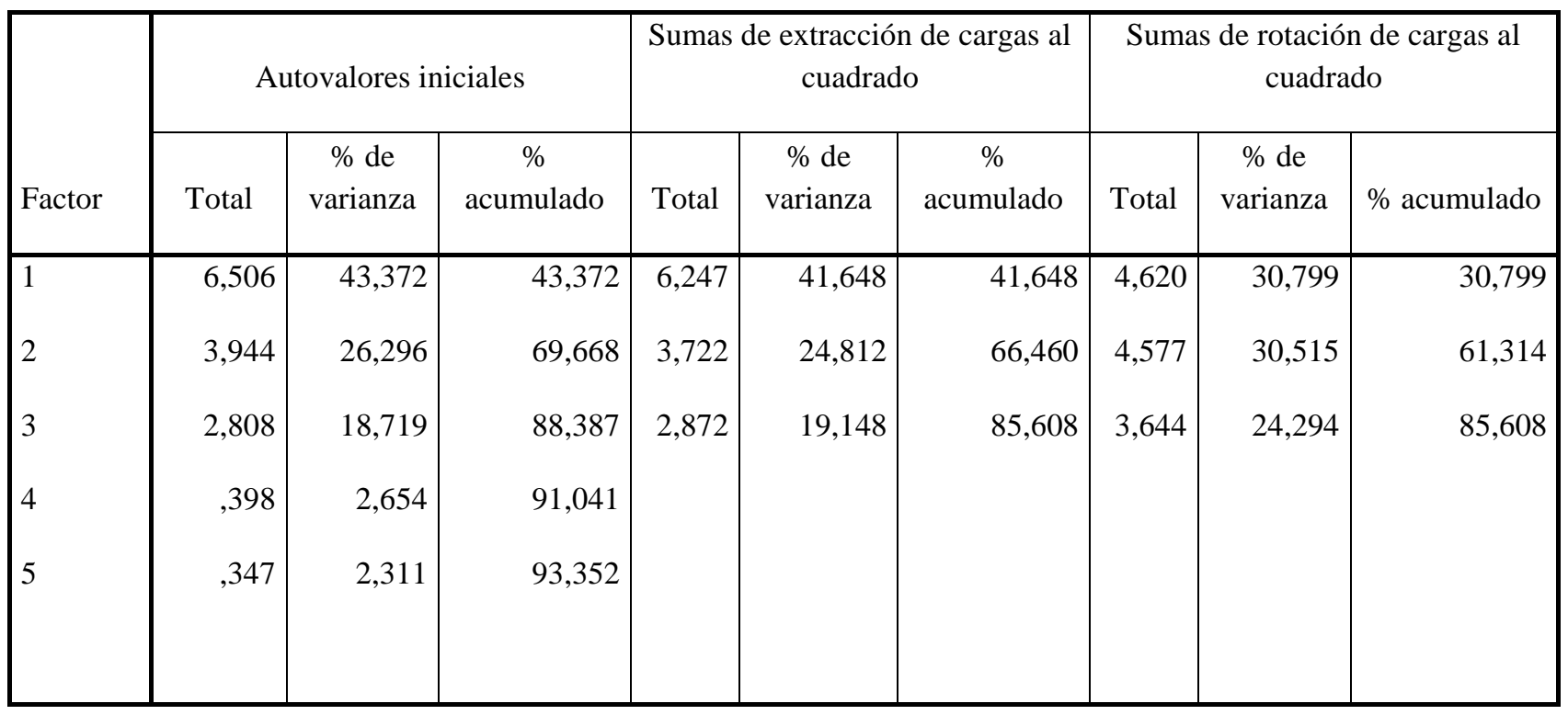

Método de extracción: máxima probabilidad. 
Análisis factorial concluyente

La rotación del modelo se aplicó a partir del procedimiento Varimax que opera con la maximización de las varianzas en las que se agrupan los nuevos factores.

Tabla No5. Matriz de factor rotado ${ }^{a}$

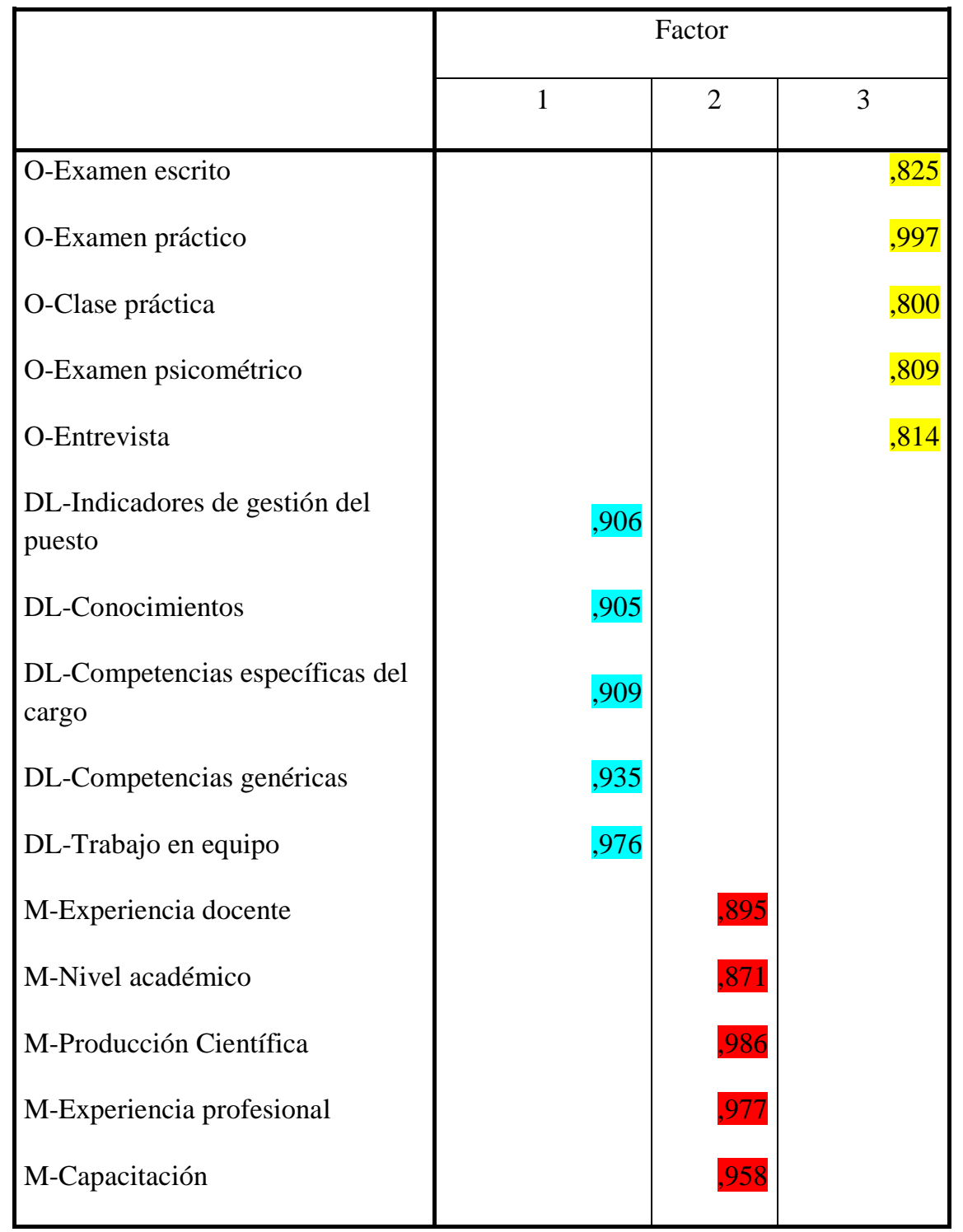

Método de extracción: máxima probabilidad.

Método de rotación: Varimax con normalización Kaiser.

a. La rotación ha convergido en 5 iteraciones. 


\section{Discusión .}

Se evidencian 3 grupos perfectamente diferenciados por la variante discriminante y dentro de cada grupo una fuerza factorial considerable que demuestra una validez de convergencia. Para concluir con el examen es pertinente observar el modelo desde un punto de vista integral. El análisis de ecuaciones estructurales permite el cálculo simultáneo de las relaciones estructurales del modelo relacional exógeno-endógeno. Dicho sea de paso, genera una valoración de sus componentes a partir de las covarianzas, esta relación permite el decremento de los errores de medida sobre la variable. Se presenta a continuación el modelo a partir del sistema estadístico Lisrel.

\section{Gráfico No1 Red Lab.}

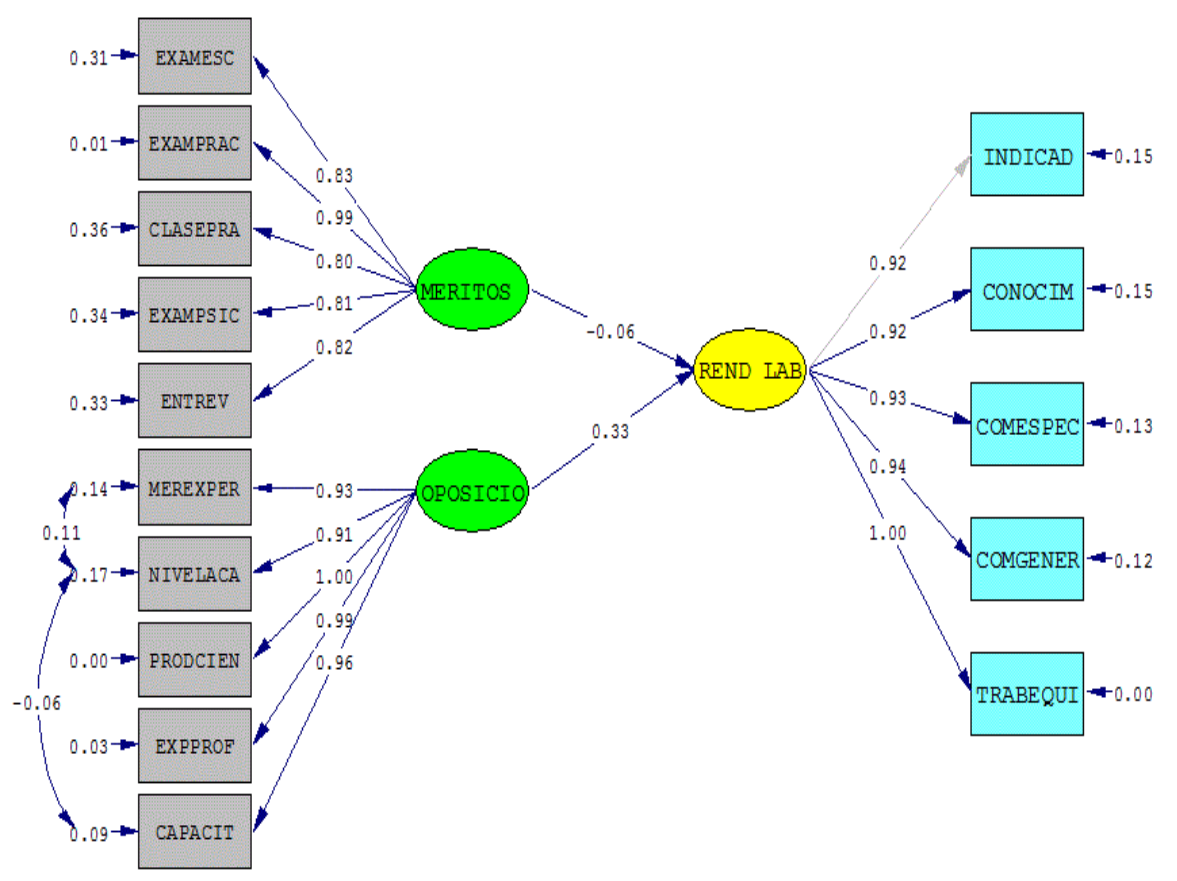

\section{Conclusiones •}

- Se evidencia una marcada tendencia de los colectivos científicos sobre la influencia de las variables de selección de personal en el rendimiento laboral. La selección de personal, aumenta la eficiencia al atraer personal más adecuado para cubrir plazas especializadas al momento del enriquecimiento del puesto. Esta investigación se basa en la operalización del experimento de Edwards y Smith que abarca dos dimensiones a decir: merecimientos y oposición. Se evidencia una influencia fuerte de los procesos de oposición sobre el rendimiento laboral. El proceso de selección por méritos influye negativamente sobre el rendimiento laboral. 


\section{Referencias bibliográficas.}

Deem, R. (2001). Globalisation, new managerialism, academic capitalism and entrepreneurialism in universities: is the local dimension still important? . Comparative Education, 7-20.

Edwards, D., y Smith, T. (2010). 'Supply issues for science academics in Australia: now and in the future'. Higher Education, 19-32.

Gilliot, D., Overlaet, B., y Verdin, P. (2002). Managing academic personnel flow at universities. Tertiary Education and Management, 277-295.

Hiltrop, j. (1999). The quest for the best: human resource practices to attract and retain talent. European Management Journal, 422-430.

Holland, P., Sheehan, C., y De Cieri, H. (2007). Attracting and retaining talent: exploring human resources development trends in Australia. Human Resource Development International, 247-262.

Huisman, J., Weert, E., y Bartelse, J. (2002). Academic careers from a European perspective: the declining desirability of the faculty position. The Journal of Higher Education, 141-160.

Janssens, M., y Steyaert, C. (2009). HRM and performance: a plea for reflexivity in HRM studies . Journal of Management Studies, 143-155.

Latham, G., Almost, J., y Moore, C. (2005). New developments in performance management. Organizational Dynamics, 77-87.

Lorange, P. (2006). A performance based, minimalist human resource management approach in business schools. Human Resource Management, 649-658.

Lubbe, V., y Larsen, V. (2008). Quick-Scan Jong Talent in De Wetenschap. Probleemanalyse Ten Behoeve Van Het Programma 'Loopbaanperspectief Jong Talent', $243-255$.

Marsh, T. (2011). Serious games continuum: Between games for purpose and experiential environments for purpose. Entertain- ment Computing.

McKenna, S., Richardson, J., y Manroop, L. (2010). Alternative paradigms and the study and practice of performance management and evaluation. Human Resource Management Review, 148-157. 
McKenna, S., Richardson, J., y Manroop, L. (2010). Alternative paradigms and the study and practice of performance management and evaluation. Human Resource Management Review, 148-157.

Metcalf, H., Rolfe, H., Stevens, P., y Weale, M. (2005). Recruitment and Retention of Academic Staff in Higher Education, Nottingham: . National Institute of Economic and Social Research, 123 - 145.

Ryan, A., y Ployhart, R. (2014). A century of selection. Annual Review of Psychology, 20.1-20.25.

Schatzki, T., Knorr Cetina, K., y Von Savigny, E. (2001). The Practice Turn in Contemporary Theory. London: Routledge., 50 - 63.

Van Balen, B., y Van den Besselaar, P. (2007). Universitaire Onderzoeksloopbanen. . Academic Careers. An Exploration of Problems and Solutions, 186 - 239.

Watson, T. (2004). HRM and critical social science analysis. Journal of Management Studies, 447-467.

Watson, T. (2010). Critical social science, pragmatism and the realities of HRM. The International Journal of Human Resource Management, 915-931.

Yanow, D. (2006). Talking about practices: on Julian Orr's Talking About Machines. Organization Studies, 1743-1756.

Yu, K., y Cable, D. (2012). Recruitment and competitive advantage: A brand equity perspective. The Oxford handbook of industrial- organizational psychology, 197-220.

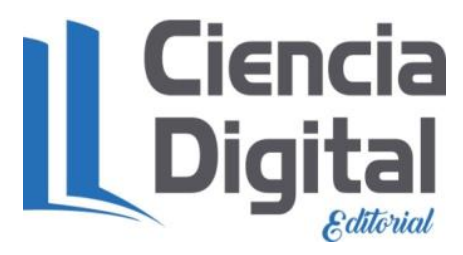


Para citar el artículo indexado.

Saltos J., Reyes L. \& Reyes J. (2018). La selección de personal como contribuyente causal del desempeño laboral, un estudio explicativo realizado a docentes universitarios de la República del Ecuador. Revista electrónica Ciencia Digital 2(2), 604-620. Recuperado desde:

http://cienciadigital.org/revistacienciadigital2/index.php/CienciaDigital/article/view/129/11 3

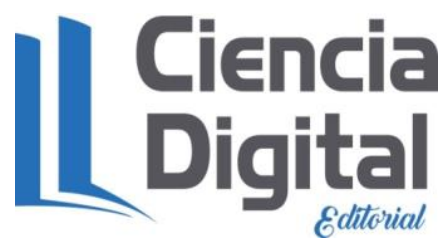

El artículo que se publica es de exclusiva responsabilidad de los autores y no necesariamente reflejan el pensamiento de la Revista Ciencia Digital.

El articulo queda en propiedad de la revista y, por tanto, su publicación parcial y/o total en otro medio tiene que ser autorizado por el director de la Revista Ciencia Digital.
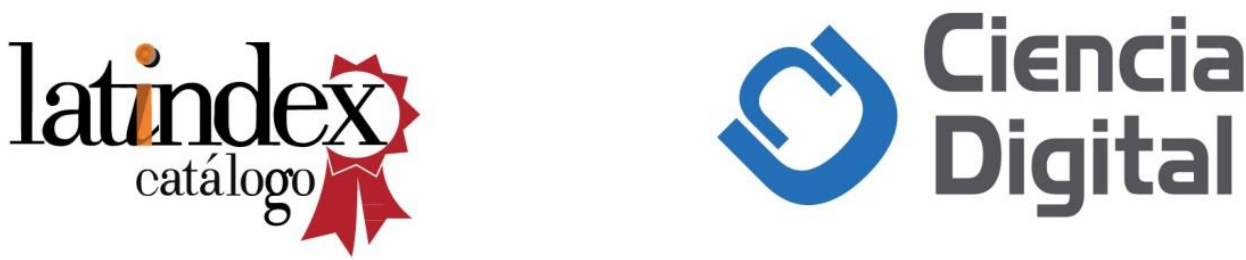